\title{
Preliminary evidences for recruitment of innate responses to rectal cancer cell death elicited by neoadjuvant radio-chemotherapy
}

\author{
A. TAMBURINI ${ }^{1}$, A. CASTIGLIONI ${ }^{2}$, M.C. SALANDINI ${ }^{1}$, K. BENCARDINO $^{3}$, E. ORSENIGO ${ }^{1}$, \\ S. DI PALO ${ }^{1}$, L. ALBARELLO ${ }^{4}$, M. RONZONI ${ }^{3}$, A. MANFREDI $^{2}$, C. STAUDACHER ${ }^{1}$ \\ ${ }^{1}$ Department of Surgery, "Vita-Salute San Raffaele" University, Milan (Italy) \\ 2 Clinical Immunology Laboratory and Tumor Gene Therapy Laboratory, Cancer Immunotherapy and Gene Therapy Program (CIGTP), Milan (Italy) \\ ${ }^{3}$ Department of Oncology, "San Raffaele" Scientific Institute, Milan (Italy) \\ ${ }^{4}$ Department of Pathology, "Vita-Salute San Raffaele" University, Milan (Italy)
}

SUMMARY: Preliminary evidences for recruitment of innate responses to rectal cancer cell death elicited by neoadjuvant radiochemotherapy.

A. Tamburini, A. Castiglioni, M.C. Salandini, K. Bencardino, E. Orsenigo, S. Di Palo, L. Albarello, M. Ronzoni,

A. MANFredi, C. STAUdACHER

Introduction: Rectal cancer patients with a cT3N+MO tumor stage responds to the neo-adjuvant therapy, which causes necrosis and inflammation in situ. We cannot predict which patients will response.

Methods: We collected blood and tissue samples at three time points: at diagnosis, at the end of the first CT cycle and at 8 week after the end of the therapy (coinciding with the surgical resection time of the tumour). At each time point we characterized circulating monocytes by flow cytometry, infiltrating macrophages by immunohistochemistry and selected inflammatory molecules in serum and plasma.

Results: We recruited 28 pts, with No substantial changes were detectable in the number of circulating monocytes. In contrast we observed a clear expansion of CD14/CD86 and CD14/CD163 double positive subsets. In fact we observed that in the responder patients the expansion of the CD14/86 subset was clear in the first weeks of treatment and decreased there after. The immunohistochemical study revealed a massive tumoral infiltration by macrophages that displayed clear features of alternative M2 polarization.

Conclusion: These data suggest that neoadjuvant therapy modulates the cellular components of innate immune responses that could represent valuable predictive factors.

KEY WORDS: rectal cancer, neoadjuvant therapy, immune responses.

\section{Introduction}

Colorectal cancer is the fourth cancer in the world with 1.023 .000 new cases and 529.000 death. Rectal cancer patients with a cT3N+M0 tumor stage responds to the neo-adjuvant therapy, which causes necrosis and inflammation in situ. We cannot predict which patients will response. We focused our attention on macrophages, which represent specialized sensors of injury in the midst of living tissues; in particular we assessed the expression of Heme Oxygenase (HO-1), CD68, CD163, CD206,

\section{Corrispondenza Autore:}

Dott. Andrea Tamburini

Dipartimento di Chirurgia Università Vita-Salute San Raffaele

Via Olgettina 60 - 20132 Milano MI

E-mail: tamburini.andreamarco@ hsr.it

(C) Copyright 2009, CIC Edizioni Internazionali, Roma
Tie2, RAGE. Moreover, we assessed inflammatory molecules and soluble pattern recognition receptors.

\section{Methods}

We collected blood and tissue samples at three time points: at diagnosis, at the end of the first CT cycle and at 8 week after the end of the therapy (coinciding with the surgical resection time of the tumour). At each time point we characterized circulating monocytes by flow cytometry, infiltrating macrophages by immunohistochemistry and selected inflammatory molecules in serum and plasma.

\section{Results}

We recruited 28 pts, with so far five complete pathological remission, five partial responses and five no responses. No substantial changes were detectable in the number of circulating monocytes. In contrast we observed a clear expansion of CD14/CD86 and CD14/ CD163 double positive subsets. This event was transient; it abated at the later time point suggesting a causal relationship to the treatment. It correlated with sensitivity to the treatment. In fact we observed that in the responder patients the expansion of the CD14/86 subset was clear in the first weeks of treatment and decreased there after. In contrast in non-responder patients it was already expanded before the neo-adjuvant therapy. All the patients had an initial expansion of the CD14/163 subset. In the responder patients this population was still present at the time of surgery. The immunohistochemical study revealed a massive tumoral infiltration by macrophages that displayed clear features of alternative M2 polarization.

\section{Conclusion}

These data suggest that neoadjuvant therapy modulates the cellular components of innate immune responses that could represent valuable predictive factors. 